\section{Dangers of asteroid deflection}

SIR - There are thought to be about 1,000 Earth-crossing asteroids, each with a diameter $>1.5 \mathrm{~km}$, although only about 50 have so far been identified ${ }^{1}$. A collision of one of these asteroids with the Earth would release an energy equivalent to $10^{5}$ MT, disrupt the ecosphere, terminate agriculture, and kill a significant fraction of the world's human population (see, for example, ref. 2). The a priori risk of such a catastrophe during the next century is about one in 5,000.

The proposed 'Spaceguard' survey ${ }^{2,3}$ could, in 25 years, find about $95 \%$ of these potentially threatening asteroids. We can predict with $>99.9 \%$ certainty that no object will be found on a trajectory posing any danger during at least the next century, in which case Spaceguard would seem to reduce the a priori risk of a global impact catastrophe by nearly two orders of magnitude. In the improbable case that an asteroid were found on a threatening trajectory, the most likely warning time would be several decades or more, long enough to mitigate the hazard. The most efficient approach with existing technology would be to deflect the object through a herding series of standoff nuclear explosions ${ }^{4,5}$.

This proposal is a double-edged sword. If we can perturb an asteroid out of impact trajectory, it follows that we can also transform one on a benign trajectory into an Earth-impactor. For example, the asteroid 1991 OA could in 2070 be deflected into Earth-impact trajectory ${ }^{5}$ with an aggregate yield of only about $60 \mathrm{MT}$. Although a single asteroid can more readily be deflected away from, than into, an impact trajectory, there is not an ordersof-magnitude difference in technical effort; but there are orders-of-magnitude more Earth-crossing asteroids that can be induced to impact the Earth than will do so on their own. With a Spaceguard-like inventory of such asteroids and a launchready deflection system of nuclear-armed missiles, it might take only a few years to identify a suitable large asteroid, alter its orbit through a series of nuclear explosions with individual yields of about 10 MT (available in existing arsenals), and send it crashing into Earth ${ }^{5}$. There is no ather way known in which a few nuclear weapons could by themselves threaten the global civilization.

In our view, development of this asteroid-deflection technology would be premature. Given twentieth-century history and present global politics, it is hard to imagine guarantees against eventual misuse of an asteroid deflection system commensurate with the dangers such a system poses. Those who argue that it would be prudent to prevent catastrophic impacts with annual probabilities of $10^{-5}$ will surely recognize the prudence of preventing more probable catastrophes of comparable magnitude from misuse of a potentially apocalyptic technology.

It is of course sensible to seek costeffective reduction of risks from all hazards to our civilization - even lowprobability hazards, of which many may remain unidentified. At a total cost of some $\$ 300$ million, Spaceguard arguably constitutes a reasonable measure of defence against the impact hazard. But premature deployment of any asteroid orbitmodification capability, in the real world and in light of well-established human frailty and fallibility, may introduce a new category of danger that dwarfs that posed by the objects themselves.

\section{Carl Sagan}

Laboratory for Planetary Studies,

Cornell University,

Ithaca, New York 14853, USA

Steven J. Ostro

Jet Propulsion Laboratory,

Pasadena, California 91109, USA

1. Shoemaker, E. M., Wolfe, R. F. \& Shoemaker, C. S. in Global Catastrophes in Earth History (eds Sharpton, V. L. \& Ward, P. D.) 155-170 (Geol. Soc. Am. Pap. 247, 1990).

2. Chapman, C. R. \& Morrison, D. Nature 367, 33 (1994).

3. Morrison, D. Acta astronautica 30, 11-16 (1993).

4. Ahrens, T. J. \& Harris, A. W. Nature 360, 429-433 (1992)

5. Harris, A. W., Canavan, G. H., Sagan, C. \& Ostro, S. J. in Hazards Due to Comets and Asteroids (ed. Gehrels, T.) (University of Arizona Press, Tucson, in the press).

\section{Statistical scrotal effect}

SIR - Nieschlag et al. ${ }^{1}$ used historical data to examine the hypothesis that testosterone influences longevity in men. They examined mean life span in 50 castrati, famous as singers during the period 1581 1858 , compared to the mean life span of 50 similarly famous 'intact' singers born at the same times. The difference in mean life span $(1.2 \mathrm{yr})$ was not significant in a two-tailed $t$-test $(P=0.65)$, leading the authors to conclude: "These data show that prepubertal removal of the testes had no influence on the longevity of men." But the authors have fallen into a very common statistical trap - they conclude that a failure to demonstrate an effect implies that the effect does not exist, that an unrejected null hypothesis is true. A more reasonable conclusion from their data would have been that there is no evidence that the mean differences are not equal, which is not the same thing as concluding that the means are equal.

It is instructive to examine this data set from a power-analysis point of view. Let us assume, as the authors did, that (1) age at death is normally distributed (this is almost certainly untrue); (2) the variances of age at death in castrati and intact singers are equal and estimable from the sample variances; and (3) the appropriate null hypothesis is that the mean age at death is the same (given the authors' preamble, a one-tailed hypothesis would have been more appropriate). Then, if the real difference in age at death were $1.2 \mathrm{yr}$, what is the probability (power) of detect-

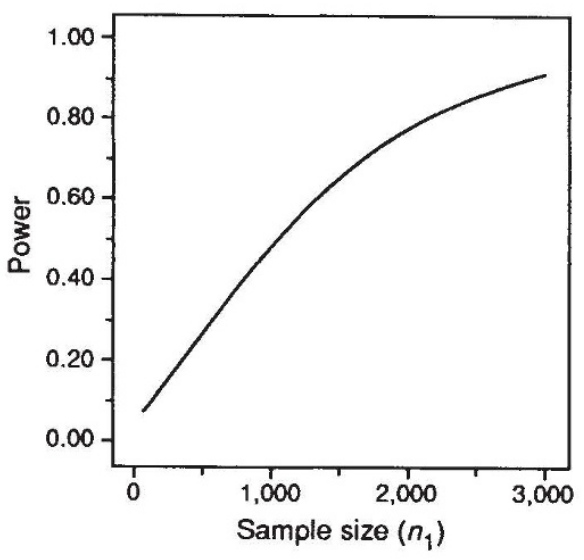

Effect of sample size on power of a two-tailed $t$-test for independent means to reject a false null hypothesis if the difference in means $=$ 1.2 yr $(\alpha=0.05, s=13.95)$.

ing such a difference with a sample of size 50 in each group? Using a pooled variance of the ages at death, the probability is only 0.071 (see figure). It is therefore not at all surprising that the authors did not detect a significant effect - they had almost no chance of doing so. If the effect of castration were to change mean lifespan (in either direction) by $1.2 \mathrm{yr}$, then, given the observed variance structure, the authors would need a sample of size $>2,120$ (of each type of singer) to have an $80 \%$ chance of detecting such an effect with a maximal probability of 0.05 of making a type-1 error. Examined in another way what is the smallest difference in mean age at death that could have been detected with this data set if the maximal probability of a type- 1 error was 0.05 and we wanted an $80 \%$ chance of detecting such a minimal difference if it existed? The answer is $9.1 \mathrm{yr}$. If the difference in longevity were that large, we probably wouldn't be engaged in statistical arguments about its validity!

The best way, of course, to conclude that a null hypothesis is probably true is by replication; if repeated attempts to falsify the hypothesis always fail, then one begins to be increasingly convinced that the hypothesis is probably true. It is clearly ill advised to conclude the truth of a hypothesis based on a single sample, in the absence of a consideration of what effect size was thought to be worth detecting, or in the absence of a power analysis of the effect size that was observed. Readers wishing a gentle and informative introduction to power analysis should consult the 